\title{
多晶铈掺杂硅酸镥闪伢陶瓷的制备和发光性能
}

\author{
范灵聪, 施 鹰, 谢建军 \\ (上海大学 材料科学与工程学院, 上海 200444)
}

摘 要: 本工作对铈离子掺杂多晶硅酸镥(LSO:Ce)闪秌材料的制备方法进行了系统研究。将 LSO:Ce 前驱体溶胶喷 雾干燥后得到了球形 LSO:Ce 前驱粉体, 该前驱粉体在 $1000^{\circ} \mathrm{C}$ 和 $1100^{\circ} \mathrm{C}$ 的温度下制烧后分别得到了不同晶型的的 单相 LSO : Ce 球形粉体。显微结构观察显示: 粉体颗粒的平均直径约为 $2 \mu \mathrm{m}$, 是由几十纳米大小的 LSO:Ce 纳米 晶粒堆积而成。A 型球形 $\mathrm{LSO}: \mathrm{Ce}$ 粉体经 $1200^{\circ} \mathrm{C} / 80 \mathrm{MPa}$ 的放电等离子体烧结(SPS)后获得了平均晶粒尺寸为 $1.3 \mu \mathrm{m}$, 相对密度高达 99.7\%的 LSO:Ce 闪伢陶瓷。由 A 型球形 LSO:Ce 粉体压制的素坏在 $1650^{\circ} \mathrm{C}$ 的空气气氛下烧结 $4 \mathrm{~h}$ 后 可获得相对密度达 $98.6 \%$, 平均晶粒尺寸为 $1.6 \mu \mathrm{m}$ 的 LSO:Ce 陶瓷。该陶瓷经 $1650^{\circ} \mathrm{C} / 150 \mathrm{MPa}$ 的热等静压(HIP)处 理 $1 \mathrm{~h}$ 后, 获得了相对密度为 99.9\%的半透明 LSO:Ce 闪炼陶瓷，其平均晶粒尺寸为 $1.7 \mu \mathrm{m}$, 晶界干净。该 LSO:Ce 陶瓷的光产额可达 28600 photons $/ \mathrm{MeV}$ ，发光衰减时间为 $25 \mathrm{~ns}$ 。

关 键 词: 硅酸锚; 制备; SPS; HIP; 闪伢陶瓷

中图分类号: TQ174 文献标识码: A

\section{Fabrication and Luminescent Property of Polycrystalline Cerium-doped Lutetium Oxyorthsilicate Scintillation Ceramics}

\author{
FAN Ling-Cong, SHI Ying, XIE Jian-Jun
}

(School of Materials Science and Engineering, Shanghai University, Shanghai 200444, China)

\begin{abstract}
Fabrication processing of cerium-doped lutetium oxyorthsilicate $\left(\mathrm{Lu}_{2} \mathrm{SiO}_{5}: \mathrm{Ce}^{3+}\right.$, $\left.\mathrm{LSO}: \mathrm{Ce}\right)$ scintillation materials was systematically investigated in this article. Spherical LSO:Ce precursor powder was fabricated through spray drying from the synthetic LSO:Ce precursor sol. Single-phased LSO:Ce spherical powders with A-type and B-type crystal structure were achieved under the calcining temperature of $1000^{\circ} \mathrm{C}$ and $1100^{\circ} \mathrm{C}$ starting from the spherical LSO:Ce precursor powder, respectively. The LSO:Ce powders with the diameter of about $2 \mu \mathrm{m}$ are solid spherical granules, which are assembled from a set of nano-sized grains. The LSO:Ce ceramics with the average grain size of $1.3 \mu \mathrm{m}$ were sintered by spark plasma sintering (SPS) under sintering temperature of $1200^{\circ} \mathrm{C}$ and the applied pressure of $80 \mathrm{MPa}$ starting from fabricated A-type LSO:Ce spherical powder. Relative density of the sintered LSO:Ce ceramics can be attained to $99.7 \%$. The pressureless sintered ceramics with relative density of $98.6 \%$ was obtained through pressureless sintering at $1650^{\circ} \mathrm{C}$ for $4 \mathrm{~h}$ in air starting from the green compact dry-pressured from an A-type LSO:Ce spherical powder. The average grain size of the pressureless sintered ceramics is $1.6 \mu \mathrm{m}$. After HIPing at $1650^{\circ} \mathrm{C}$ under an argon atmosphere for $1 \mathrm{~h}$, a translucent LSO:Ce ceramics with clear grain boundary was achieved. The relative density of the HIPed ceramics is $99.9 \%$ and its average grain size is $1.7 \mu \mathrm{m}$. The light yield of the HIPed LSO:Ce ceramics reaches 28600 photons $/ \mathrm{MeV}$ and its luminescence decay time is $25 \mathrm{~ns}$.
\end{abstract}

收稿日期: 2017-07-03; 收到修改稿日期：2017-11-20

基金项目：国家自然科学基金(51172139, 11079026) National Natural Science Foundation of China $(51172139,11079026)$

作者简介: 范灵聪(1986-), 男, 讲师. E-mail: lcfan@shu.edu.cn

通讯作者: 施 鹰, 研究员. E-mail: yshi@shu.edu.cn 
Key words: lutetium oxyorthosilicate; fabrication; SPS; HIP; scintillation ceramics

铈掺杂硅酸锚 $\left(\mathrm{Lu}_{2} \mathrm{SiO}_{5}: \mathrm{Ce}^{3+}, \mathrm{LSO}: \mathrm{Ce}\right)$ 是一种综 合性能优良的高性能闪炼材料, 主要应用于核医学 成像领域。从其本征特性来看, LSO:Ce 的密度高达 $7.40 \mathrm{~g} / \mathrm{cm}^{3}$, 折射率仅为 1.81。LSO:Ce 的光产额高 达 27300 photons $/ \mathrm{MeV}$; 闪炼衰减时间仅为 $40 \mathrm{~ns}$; 能 量分辨率约为 $9 \%$; 发光峰位 $(420 \mathrm{~nm})$ 与 PMT 敏感 区非常匹配。辐照长度为 $1.14 \mathrm{~cm}$; 抗辐照硬度高, 在辐射剂量为 $10^{6}$ 拉德( $\left.\mathrm{rad}\right)$ 时不会出现损伤, 在剂 量达 $10^{8} \mathrm{rad}$ 时表现出微小的损伤。

经过二十多年的研发, LSO:Ce 单晶生长技术取 得了一定的进展, 少数科研机构(如四川压电与声 光技术研究所、中国科学院上海硅酸盐所)和公司 (圣戈班陶瓷和塑料公司、晶体光子公司)已能生长 出大尺寸的单晶 ${ }^{[1]}$, 但是由于 Ce 在 LSO 中的分凝系 数很小 $(\sim 0.22)^{[2]}$, 造成生长的 $\mathrm{LSO}$ 晶体中 $\mathrm{Ce}^{3+}$ 离子 分布极不均匀, 在晶体底部浓度远高于顶部, 以致 于底部材料的光输出是顶部的 2 5 倍, 而闪㧎衰减 时间则从 $29 \mathrm{~ns}$ 增加到 $46 \mathrm{~ns}$, 这样所生长的晶体只 有一部分可使用 ${ }^{[3-4]}$ 。而能量分辨率的变化则更 大, 不同实验室所测值均有差异, 可从 $7 \%$ 变化到 $17 \%^{[1,5-7]}$ 。此外, LSO 的熔点高达 $2150^{\circ} \mathrm{C}$, 如此高的 生长温度对生长设备提出了巨大挑战, 主要表现在 铱坩埚中的铱特别容易挥发或被熔蚀到熔体中, 这 不仅增加了生长成本, 而且挥发出的铱金颗粒容易 进入晶体中形成光散射中心 ${ }^{[8-10]}$ 。此外, 高温导致保 温材料的损耗非常大 ${ }^{[11]}$, 也使所生长晶体热应力较 大，容易引起晶体开裂。

由于 LSO:Ce 单晶存在上述问题, 尤其是较高 的熔点和 Ce 离子在 LSO 中分布不均的问题增加了 LSO:Ce 单晶生产成本。Ce 离子在 $\mathrm{L}(\mathrm{Y}) \mathrm{SO}$ 中分布 不均的问题很难得到解决, 即使像圣戈班这样知名 的晶体生长公司在近年的报道中也表明 Ce 在 LYSO 晶体的头部和脚部的分布比例为 17:30(通过辉光放 电质谱检测 $)^{[4]}$ 。这两方面的问题使得 LSO:Ce 单晶难 以完全取代目前 PET 上广泛应用的 BGO 单晶。而 通过多晶陶瓷途径制备 LSO:Ce 闪炼体, 不但保留 了 LSO:Ce 单晶的优良性能, 还极大地降低了生产 成本 ${ }^{[12-14]}$, 这非常有利于 LSO:Ce 闪炼体进一步商 业化。本工作采用喷雾干燥法制备了球形 LSO:Ce 粉体, 在此基础上采用放电等离子体烧结(SPS)和 热等静压(HIP) 技术制备了 LSO:Ce 陶瓷, 并对 LSO:Ce 陶瓷的显微结构、发光和闪炼性能进行了系 统研究。

\section{1 实验方法}

\section{1 球形 LSO:Ce 粉体制备}

以 $\mathrm{LuCl}_{3} \cdot 6 \mathrm{H}_{2} \mathrm{O}$ (纯度 $99.99 \%$ )、 $\mathrm{CeCl}_{6} \cdot 7 \mathrm{H}_{2} \mathrm{O}$ (纯度 99.9\%)和正硅酸四乙酯(分析纯)为原料, 异丙醇(分 析纯)为液相介质, 环氧丙烷(分析纯)为反应助剂, 采用溶胶-凝胶法制备了分散的溶胶 ${ }^{[15]}$, 其中 $\mathrm{Lu}$ 、 Ce 原子比为 $200: 1$ 。处于搅拌中的液态溶胶被蠕动 泵吸入小型实验喷雾干燥机的喷嘴, 随后被 $0.3 \mathrm{MPa}$ 的空气压入干燥室中, 再被同方向 $150^{\circ} \mathrm{C}$ 热空气干 燥。干燥后的大颗粒粉体落入干燥室下方的集料瓶 中, 小颗粒物料则随热空气一起进入旋风分离器后 被细颗粒集料瓶所收集。所收集到的细颗粒前驱粉 体，在 $1000 \sim 1300^{\circ} \mathrm{C}$ 的空气氛下煅烧 $2 \mathrm{~h}$ 。

\subsection{SPS 烧结 LSO:Ce 陶瓷}

由于 SPS 烧结采用高密度脉冲电流直接对模具 和粉体颗粒加热, 热效率非常高, 升降温速率极其 迅速，在温度和压力的共同作用下，粉体颗粒的塑 性流动显著增加, 物质迁移明显变快, 根据烧结样 品的大小可在几十分钟内就可以完成一炉样品的烧 结过程。通常 LSO:Ce 陶瓷在无压烧结条件下需要 在高温下保温数小时才能达到致密 ${ }^{[16]}$, 而采用 SPS 烧结, 只需几分钟就能完成致密化过程 ${ }^{[17]}$ 。

为了便于脱模和减少陶瓷渗碳, 首先将一层喷 涂有 BN 粉体的碳纸垫入石墨模具的内壁, 然后在 石墨模具中装填 $2.5 \mathrm{~g} \mathrm{LSO}$ :Ce 粉体(炦烧温度为 $\left.1000^{\circ} \mathrm{C}\right)$, 最后将模具放置于 SPS 烧结炉中烧结。在 SPS 烧结过程中, 炉体内气压小于 $10 \mathrm{~Pa}$, 在 $900^{\circ} \mathrm{C}$ 以下的升温速率为 $85^{\circ} \mathrm{C} / \mathrm{min}, 900^{\circ} \mathrm{C}$ 以上的升温速率 为 $60^{\circ} \mathrm{C} / \mathrm{min}$, 烧结温度为 $1150 \sim 1350^{\circ} \mathrm{C}$, 最大压力 为 $80 \mathrm{MPa}$, 保温时间为 $5 \mathrm{~min}$ 。

\subsection{HIP 烧结 LSO:Ce 陶瓷}

通常情况下, HIP 烧结通过惰性气体向材料的 各个方向上均匀地传递压力, 材料中的晶界在高温 和高压相互作用下产生滑移, 从而使材料晶界处的 残余气孔被有效地排出, 使材料达到高度致密化。 陶瓷相对密度的提高依赖于选择合适的预烧结温度 和 HIP 后处理温度。

陶瓷材料通常需要采用无压烧结排出材料中的 开口气孔后再进行 HIP 烧结。在 $1000^{\circ} \mathrm{C}$ 空气氛下炦 烧后获得的 LSO:Ce 粉体经干压(20 MPa)和冷等静 压(200 MPa)后获得相对密度为 53\%的 LSO:Ce 素 
坏。LSO:Ce 素坏在 $1650^{\circ} \mathrm{C}$ 的空气氛下烧结 $4 \mathrm{~h}$ 后得 到相对密度为 $98.6 \%$ 的陶瓷, 再将该陶瓷进行 HIP 烧结。烧结程序如下: 在 $300^{\circ} \mathrm{C}$ 以下采用手动升温, 氩气的起始压力为 $52 \mathrm{MPa} ; 300^{\circ} \mathrm{C}$ 以上采用程序升 温, 300 至 $900^{\circ} \mathrm{C}$ 的升温速率为 $13^{\circ} \mathrm{C} / \mathrm{min}$, 随后以 $6^{\circ} \mathrm{C} / \mathrm{min}$ 的升温速率达到 $1650^{\circ} \mathrm{C}$, 气体的压力随温 度的升高而增大, 此时的压力为 $150 \mathrm{MPa}$ 左右, 在 此温度和压力条件下停留 $1 \mathrm{~h}$ 后随炉冷却。最后将 HIP 烧结后的 LSO:Ce 陶瓷在 $1300^{\circ} \mathrm{C}$ 的空气气氛下 退火 $4 \mathrm{~h}$ 以消除 HIP 烧结过程中由于强还原气氛(石 墨发热体和石墨坩埚)导致的氧空位。

\section{4 样品表征}

采用德国耐驰仪器公司的 STA409PC 型综合 热分析仪对 LSO:Ce 前驱粉体进行热重-差热(Thermal Gravity-Differential Scanning Calorimeter, TGDSC)分析。采用日本理学(Rigaku)的 D/MAX2200V PC 型 $X$ 射线衍射仪采集粉体的 $X$ 射线衍射 (X-ray diffraction, XRD)谱以分析其物相。以铜靶在 $40 \mathrm{kV}$ 电压, $40 \mathrm{~mA}$ 电流下产生的 $\mathrm{K} \alpha$ 射线照射样品, 连续 扫描样品的速度为 $2 \theta=8 \% / \mathrm{min}, 2 \theta$ 的范围为 $10 \sim$ $80^{\circ}$ 。采用荷兰 Philips 公司的 XL-30 型热场发射扫 描电子显微镜(Scanning Electron Microscope, SEM) 观察粉体和陶瓷的显微结构。为了观察 LSO:Ce 陶瓷 的表面形貌, 将陶瓷经过粒度为 $0.1 \mu \mathrm{m}$ 的金刚石抛 光亳抛光后在 $1350^{\circ} \mathrm{C}$ 的空气气氛下热腐蚀 $4 \mathrm{~h}$ 。

采用日本岛津制作所(Shimadzu)的 RF-5301PC 型苂光光谱仪测试 LSO:Ce 陶瓷的光致发射(Photoluminescence Emission, PL) 谱和光致激发 (Photoluminescence Excitation, PLE)谱。LSO:Ce 陶瓷的选 区阴极射线激发发光(Cathode Luminescence, CL)谱, 采用美国 FEI 公司的 400 FEG 型 SEM 表征, 该 SEM 配备有美国 Gatan 公司的 Mono CL3+型 CL 谱表征 系统。采用日本日立公司的 U2910 型分光光度计表 征 LSO:Ce 陶瓷的直线透过率。在 LSO:Ce 陶瓷及锗 酸铋(BGO)单晶样品的光产额表征过程中, 以 ${ }^{137} \mathrm{Cs}$ 为 $\gamma$ 射线激发源, 采用日本滨松光子学株式会社 (Hamamatsu)的 R2059 型 PMT 作为探测器。对于 LSO:Ce 陶瓷门宽 (gate) 值设为 $100 \mathrm{ns,}$, 对于 BGO 单 晶的 gate 值设为 $500 \mathrm{~ns}$, 两样品都采用同一 PMT 偏 压值进行测试。LSO:Ce 陶瓷的发光衰减曲线通过英 国爱丁堡仪器公司(Edinburgh Instruments)的 FLSP920 型全功能稳态/瞬态荧光光谱仪测试。该光谱仪 以脉冲氢放电闪光灯作为纳秒级激发光源, 测试范 围为 $500 \mathrm{~ns}$, 当计数到达 2000 以后测试停止。以上 发光和闪烐性能表征均在室温条件下操作。

\section{2 结果与讨论}

\section{1 差热一热重及物相分析}

LSO 存在 A 型和 B 型两种不同晶型结构。其中 A 型 LSO 是一种亚稳相结构 ${ }^{[18-20]}$ 。A 型 LSO 空间 群为 $\mathrm{P} 21 / \mathrm{c}$, 晶胞参数为 $a=0.8979 \mathrm{~nm}, b=0.6701 \mathrm{~nm}$, $c=0.6592 \mathrm{~nm}, \beta=104.221^{\circ}$, 单胞原子数 $Z=4^{[18]} \circ \mathrm{A}$ 型 LSO 沿[100]方向的平面原子结构包含有 $\left[\mathrm{OLu}_{4}\right]$ 四面 体和 $\left[\mathrm{SiO}_{4}\right]$ 四面体, 它们通过顶角连接成二维网络。 在这种结构中, $\mathrm{Lu}$ 原子有两种结晶学取向, 其配位 数分别为 9 和 7 。B 型 LSO 的晶体结构属于 C2/c 空间群, $a=1.4254 \mathrm{~nm}, b=0.6641 \mathrm{~nm}, c=1.0241 \mathrm{~nm}$, $\beta=122.2^{\circ}$, 单胞原子数 $Z=8^{[21]}$ 。在这种结构里, $\mathrm{Lu}$ 原 子也有两种结晶学取向, 其配位数分别为 7 和 6 , 任 意标记为 $\mathrm{Lu} 1$ 和 $\mathrm{Lu} 2$, 其中 $\mathrm{Lu} 1$ 与 5 个 $\left[\mathrm{SiO}_{4}\right]$ 的 $\mathrm{O}$ 和 2 个孤立的 $\mathrm{O}$ 配位形成多面体, $\mathrm{Lu} 2$ 与 4 个 $\left[\mathrm{SiO}_{4}\right]$ 的 $\mathrm{O}$ 和 2 个孤立的 $\mathrm{O}$ 配位形成赝八体, $\left[\mathrm{SiO}_{4}\right]$ 与 $\left[\mathrm{OLu}_{4}\right]$ 四面体通过共边连接。

喷雾干燥后的 LSO:Ce 前驱粉体以 $10^{\circ} \mathrm{C} / \mathrm{min}$ 的 升温速率在空气气氛下获得的 TG-DSC 曲线如图 1 所示。TG 曲线表明在 $600^{\circ} \mathrm{C}$ 以下是前驱体的主要失 重阶段, 这归因于前驱体中的有机物发生氧化和分 解, 可从 DSC 曲线对应温度段的放热峰得到证实, 温度到达 $1200^{\circ} \mathrm{C}$ 时的总质量损失为 $27 \%$ 。一个较平 缓的放热峰存在于 DSC 曲线 800 至 $1000^{\circ} \mathrm{C}$ 区间内, 这对应于前驱体晶化。 $1050^{\circ} \mathrm{C}$ 处的放热峰来源于 $\mathrm{A}$ 型 LSO 向 B 型 LSO 的相变放热。

喷雾干燥后的 LSO : Ce 前驱粉体在 $1000^{\circ} \mathrm{C}$ 和 $1100^{\circ} \mathrm{C}$ 空气氛下煅烧 $2 \mathrm{~h}$ 后的 XRD 图谱如图 2 所 示。经过 $1000^{\circ} \mathrm{C}$ 炦烧后的产物的物相为单一 $\mathrm{A}$ 型 LSO(A-LSO)相 ${ }^{[18]}$, 其衍射峰位置和相对强度与无 机晶体衍射数据库(Inorganic Crystal Diffraction

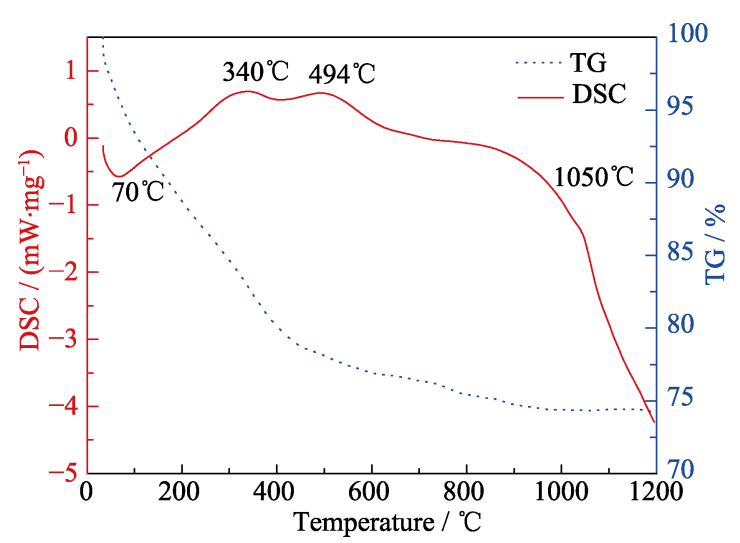

图 1 喷雾干燥后 LSO:Ce 前驱粉体的 TG-DSC 曲线 Fig. 1 TG-DSC curves of the LSO:Ce precursor derived from spray drying 

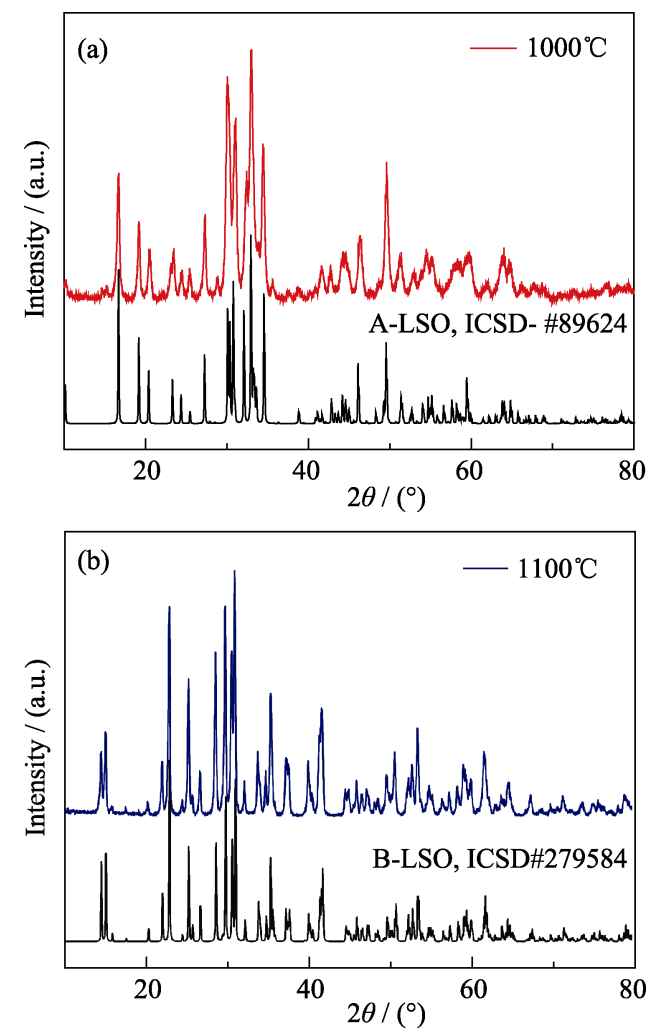

图 2 在(a) 1000 和(b) $1100^{\circ} \mathrm{C}$ 下㷽烧的粉体的 XRD 图谱

Fig. 2 XRD patterns of the precursors calcined at (a) $1000^{\circ} \mathrm{C}$ and (b) $1100^{\circ} \mathrm{C}$

Database, ICSD)中卡片号为 89624 的卡片完全匹配。 而在 $1100^{\circ} \mathrm{C}$ 㷽烧后的粉体的衍射数据与 ICSD 中 279584 号卡片数据完全吻合, 这表明在该温度下可 合成单相 B 型 LSO(B-LSO)粉体。该温度比 Wang 等所报道的 LSO 固相反应温度低 $200^{\circ} \mathrm{C}^{[22]}$, 也比其 他 LSO 液相法制备温度低 $100^{\circ} \mathrm{C}^{[23]}$ 。

\subsection{LSO:Ce 粉体形貌}

图 3 给出了喷雾干燥法制备的 LSO:Ce 前驱体 及其在各温度下㷽烧 $2 \mathrm{~h}$ 后的形貌。图 3(a)为前驱 体颗粒的低放大倍率 SEM 照片, 颗粒呈球状, 尺寸 分布窄, 分散均匀。图 3(b)中的高放大倍率 SEM 照 片表明该前驱粉体是由众多纳米小颗粒构成的, 其 单颗粒尺寸约为 $2 \mu \mathrm{m}$ 。经过 $1000^{\circ} \mathrm{C}$ 煅烧后(图 3(c)) 得到的粉体粒径仍为 $2 \mu \mathrm{m}$ 左右, 该颗粒是由晶粒 尺寸为几十纳米的晶粒组成, 表面光滑。1100 ${ }^{\circ} \mathrm{C}$ 陆烧 后的粉体晶粒尺寸呈现一定长大现象, 这可能是由 于粉体相变所致(A 相-LSO 到 B 相-LSO 的相变过程 会发生 $6.8 \%$ 的体积膨胀)。在炦烧温度由 $1100^{\circ} \mathrm{C}$ 升高 至 $1300^{\circ} \mathrm{C}$ 过程中, 粉体颗粒仍保持球状, 但粉体颗 粒尺寸呈现显著收缩，这是由于其晶粒之间出现烧 结和显著的长大所致, 在 $1300^{\circ} \mathrm{C}$ 时晶粒已经长大到 $400 \mathrm{~nm}$ 左右, 晶粒间烧结现象非常明显, 颗粒尺寸 已减小到 $1.6 \mu \mathrm{m}$ 左右。 $1300^{\circ} \mathrm{C}$ 的 SEM 照片还进

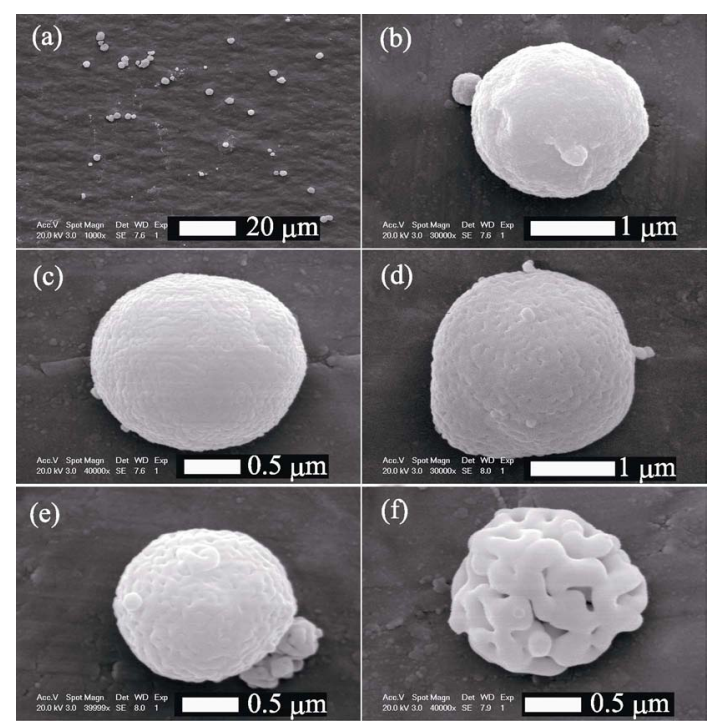

图 3 喷雾干燥法制备的前驱体(a, b)及在(c)1000、(d)1100、 (e)1200 和(f) $1300^{\circ} \mathrm{C}$ 裧烧后粉体的 SEM 照片

Fig. 3 SEM images of the precursors $(a, b)$ and precursors calcined at (c) $1000^{\circ} \mathrm{C}$, (d) $1100^{\circ} \mathrm{C}$, (e) $1200^{\circ} \mathrm{C}$ and (f) $1300^{\circ} \mathrm{C}$

一步表明所合成的球形粉体是由小颗粒密堆积成的 实心球形大颗粒。结合 XRD 图谱和 SEM 照片表明 $1000^{\circ} \mathrm{C}$ 㷽烧的 LSO:Ce 粉体最有利于后续陶瓷烧结。

\subsection{LSO:Ce 陶瓷的 SPS 烧结和显微结构}

微米级球形粉体在 $1150 \sim 1350{ }^{\circ} \mathrm{C}$ 烧结温度 (80 MPa 压力)下得到的 LSO:Ce 陶瓷 SEM 形貌如图 4 所示。在 $1150^{\circ} \mathrm{C}$ 的烧结温度下, LSO:Ce 陶瓷晶界 处存在大量孤立闭口气孔和少量连通气孔, 其平均 晶粒尺寸约为 $0.4 \mu \mathrm{m}$ 。在 $1200^{\circ} \mathrm{C}$ 的烧结温度下, 陶瓷 中的绝大部分气孔被排出, 其平均晶粒尺寸增长至 $1.3 \mu \mathrm{m}$ 。当烧结温度提高至 $1250^{\circ} \mathrm{C}$ 后, LSO:Ce 陶瓷 晶粒进一步长大, 其平均晶粒尺寸增长至 $1.6 \mu \mathrm{m}$ 。在烧 结温度升高到 $1300^{\circ} \mathrm{C}$ 后, 陶瓷晶粒尺寸增至 $2.9 \mu \mathrm{m}$ 。 由于该烧结条件 $\left(1300^{\circ} \mathrm{C} / 80 \mathrm{MPa}\right)$ 下晶界迁移速率 大于气孔迁移速率, 导致部分残余分气孔来不及排 出, 在晶粒内形成微气孔, 如图 4(d)所示。在烧结温 度进一步提高至 $1350^{\circ} \mathrm{C}$ 以后(图 4(e)), 晶界迁移速 率也进一步提高, 陶瓷晶粒发生了显著的异常长大, 其平均晶粒尺寸增大至 $40 \mu \mathrm{m}$ 以上, 大球形气孔残 留在晶界处, 在断口的晶界处还呈现长裂缝。

图 5 给出了 LSO:Ce 陶瓷的平均晶粒尺寸和气 孔率随 SPS 烧结温度的变化规律。在 $1200^{\circ} \mathrm{C}$ 所获得 的 LSO:Ce 陶瓷具有较小的平均晶粒尺寸和最低的 气孔率, 这表明这种微米级球形 LSO:Ce 粉体的最 佳 SPS 烧结温度在 $1200^{\circ} \mathrm{C}$ 附近。

\subsection{LSO:Ce 陶瓷的 HIP 烧结和显微结构}

在 $1650^{\circ} \mathrm{C}$ 的空气气氛下烧结 $4 \mathrm{~h}$ 后的 LSO:Ce 陶瓷的表面形貌如图 6(a)所示，陶瓷中的残余气孔 

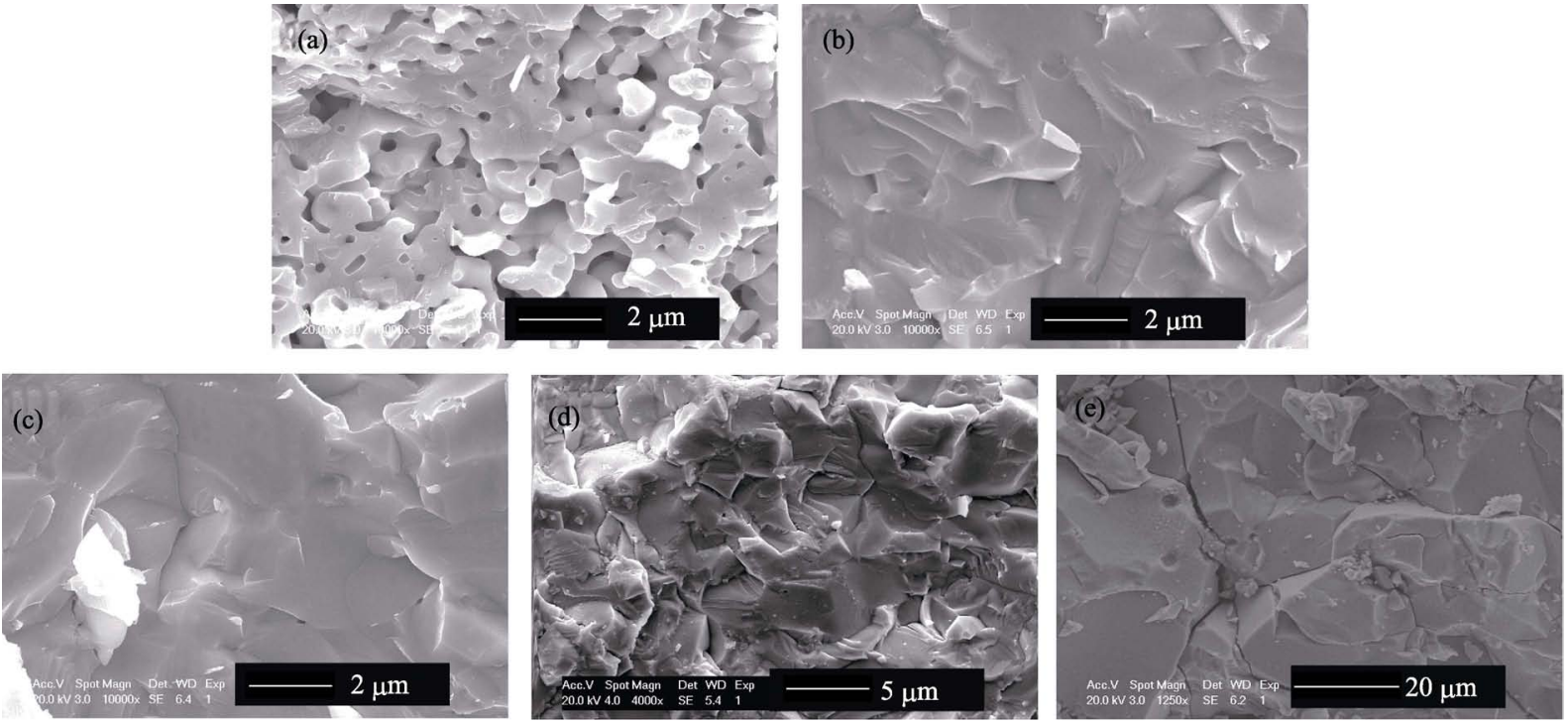

图 4 以微米级球形粉体在(a)1150、(b)1200、(c)1250、(d)1300 和(e)1350 C 温度下 SPS 烧结 5 min 的 LSO:Ce 陶瓷断面 SEM 照片

Fig. 4 SEM images of fracture surfaces of LSO:Ce ceramics SPS-sintered at (a) $1150^{\circ} \mathrm{C}$, (b) $1200^{\circ} \mathrm{C}$, (c) $1250^{\circ} \mathrm{C}$, (d) $1300^{\circ} \mathrm{C}$ and (e) $1350^{\circ} \mathrm{C}$ for 5 min from micrometer-sized spherical powders

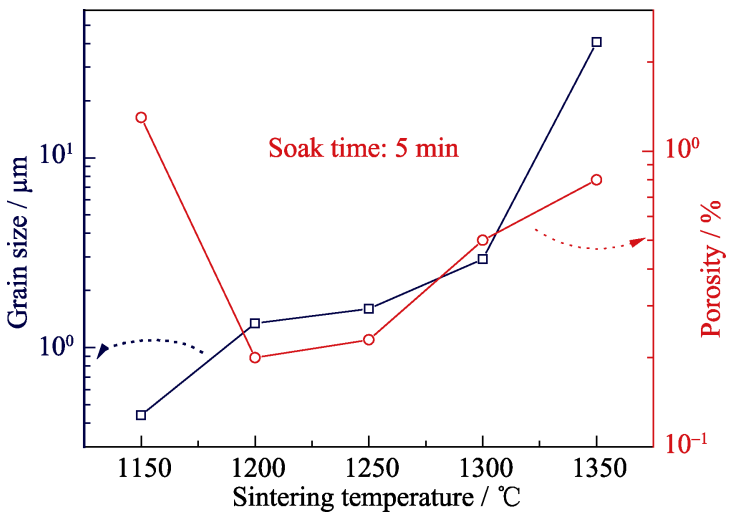

图 5 SPS 烧结温度对 LSO:Ce 陶瓷晶粒尺寸和气孔率的影响 Fig. 5 Dependence of the grain size and the porosity of SPSsintered LSO:Ce ceramics on the sintering temperature

主要以闭口气孔形式存在, 其平均晶粒尺寸为 $1.6 \mu \mathrm{m}$, 相对密度达 98.6\%, 这样的晶粒尺寸和相对密度有 利于后续 HIP 烧结。该 LSO:Ce 陶瓷在 $1650^{\circ} \mathrm{C}$, $150 \mathrm{MPa}$ 的氩气气氛下烧结 $1 \mathrm{~h}$ 后，残余气孔被完全 排出，陶瓷晶界干净，未观察到气孔和第二相，平
均晶粒尺寸为 $1.7 \mu \mathrm{m}$, 如图 6(b)所示。

\subsection{LSO : Ce 陶瓷的紫外激发发光特性和闪烁} 性能

热等静压烧结后的 LSO:Ce 陶瓷的光致激发和 发射谱如图 7 所示。在 $360 \mathrm{~nm}$ 激发下, 发射谱呈现 出一个宽发射带, 对应于 $\mathrm{Ce}^{3+}$ 的 $5 \mathrm{~d} \rightarrow 4 \mathrm{f}$ 辐射跃迁。 在 $406 \mathrm{~nm}$ 发射监测波长下, 激发谱呈现三个激发带, 分别位于 265, 300 和 $360 \mathrm{~nm}$ 处, 它们对应于 $\mathrm{Ce}^{3+}$ 的 $5 \mathrm{~d}$ 高能激发态向 $5 \mathrm{~d}$ 第一激发态发生快速无辐射 跃迁后, $5 \mathrm{~d}$ 第一激发态再向 $4 \mathrm{f}$ 能级跃迁而产生苂光 发射。

闪秌材料的发光效率强烈依赖材料的组成及其 缺陷浓度。在热压烧结的 YAG:Ce 陶瓷中小晶粒陶 瓷(平均晶粒尺寸为 $10 \mu \mathrm{m}$ )的发光强度 ${ }^{137} \mathrm{Cs}$ 照射) 仅能达到大晶粒陶瓷(平均晶粒尺寸为 $40 \mu \mathrm{m}$ ) 的 $22 \%, \mathrm{Zych}$ 等 ${ }^{[24]}$ 认为自由载流子在缺陷位置(晶界) 处无辐射跃迁和 Ce 离子发射能级与晶界处的畸变 能带相互作用而淬灭，因此小晶粒陶瓷中具有更多
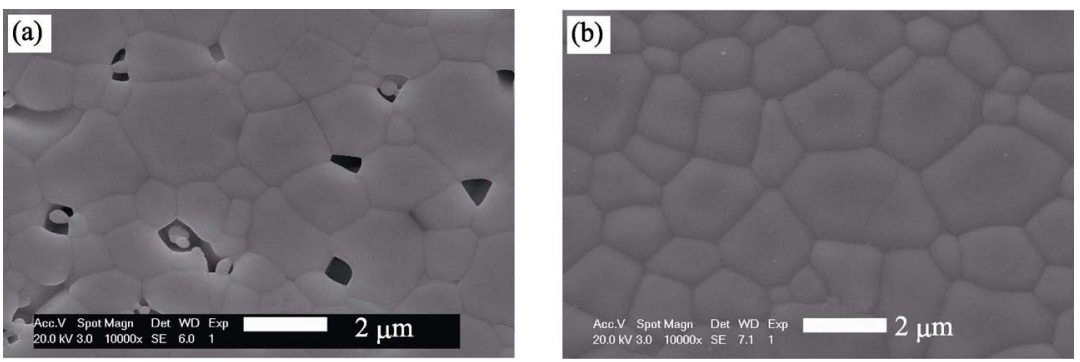

图 6 LSO:Ce 陶瓷在(a)HIP 前和(b)HIP 后的抛光表面形貌

Fig. 6 Polished surface morphologies of LSO:Ce ceramics (a) before and (b) after HIPing 


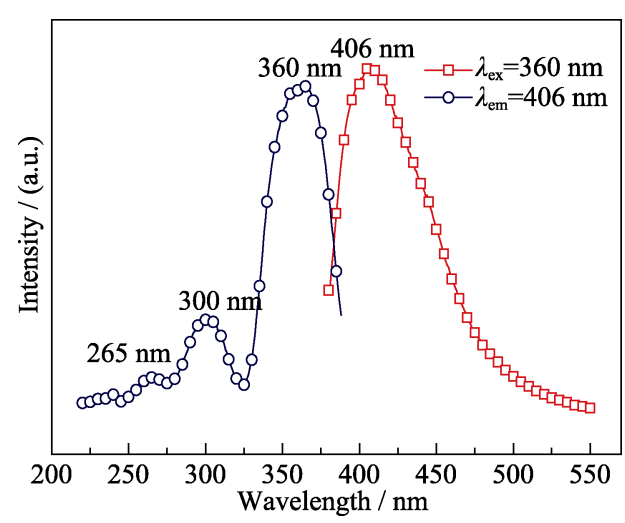

图 7 热等静压 LSO:Ce 陶瓷的光致发射和激发谱

Fig. 7 PL and PLE spectra of the HIPed LSO:Ce ceramics

的晶界会导致发光强度下降。在 $10 \mathrm{keV}$ 的电子束激 发下, LSO:Ce 陶瓷在 320 至 $600 \mathrm{~nm}$ 范围内呈现出 一个宽发射带。在该陶瓷的粗晶粒区域(晶粒尺寸为 $3.7 \mu \mathrm{m}$ ) 和细晶区域(平均晶粒尺寸为 $0.8 \mu \mathrm{m}$ ) 的 CL 发光特征非常一致(发光峰位和强度基本一致), 如 图 8 所示。这表明当陶瓷的晶粒尺寸处于 $0.8 \sim 3.7 \mu \mathrm{m}$ 范围内, 并且材料组成和缺陷浓度一致的条件下 (同一陶瓷样品), LSO:Ce 陶瓷中晶界并不会成为闪 炼材料的发光淬灭区域。实际上, 当材料的晶粒尺 寸大于 $100 \mathrm{~nm}$ 后, 处于晶界处的原子比例小于 $0.5 \%$, 晶界处晶格畸变导致的缺陷对整个晶粒发光性能的 影响也变小, 而晶粒内部的缺陷对材料的发光有更 重要的影响。Zych 等 ${ }^{[24]}$ 观察到的发光效率随晶粒减 小而减小的现象很可能来自于两种不同陶瓷的起始 粉体中不同杂质(缺陷)的影响, 而非晶界本身。

图 9 给出了 LSO:Ce 陶瓷热等静压处理后的直 线透过率, 其在 $420 \mathrm{~nm}$ (发光峰位中心)处的直线透 过率数值为 $1.4 \%$ 。图 9 中的插图表明该 LSO:Ce 闪 㷧陶瓷呈现出良好的光学半透明性。对于这样高致

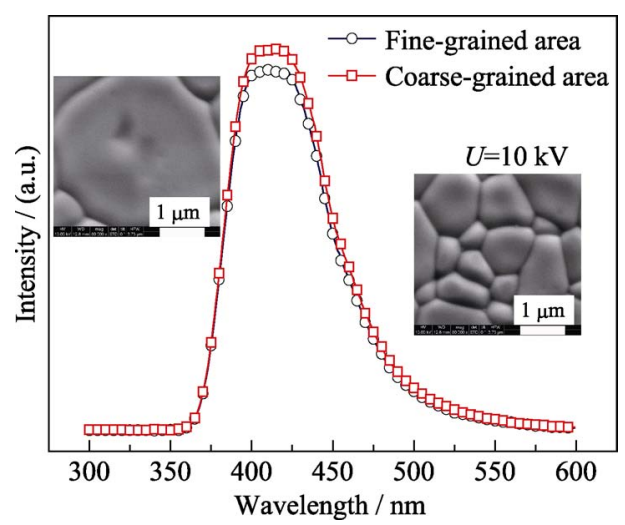

图 8 热等静压 LSO:Ce 陶瓷在阴极射线激发下的发射光谱, 插图为相应的粗晶粒区和细晶粒区形貌

Fig. 8 CL spectra of the HIPed LSO:Ce ceramics Insets are the morphologies of the respective coarse-grained and finegrained area

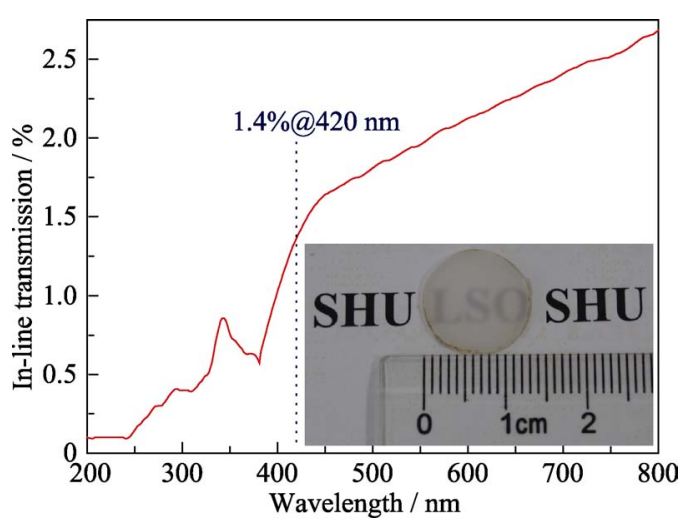

图 9 热等静压 LSO:Ce 陶瓷的直线透过率曲线, 插图为该 陶瓷的实物照片

Fig. 9 In-line transmittance curve of the HIPed LSO:Ce ceramics

Inset is photograph of the HIPed ceramics

密的单相 LSO:Ce 陶瓷, 其较低的透过率数值与 LSO 单斜晶系结构密切相关。从 LSO 的折射率数据 来看, $a$ 轴方向和 $c$ 轴方向上 LSO 的折射率差值高 达 0.028 , 这势必造成入射光通过晶粒随机取向的 多晶材料时引起强烈散射从而严重降低入射方向上 的透射光强度 ${ }^{[25-26]}$ 。

$\mathrm{BGO}$ 单晶的闪炼发光来源于 $\mathrm{Bi}^{3+}$ 本征发光, 且 抗辐照硬度高, 闪秌发光性能稳定, 室温下的光产 额为 8500 9000 ph/MeV, 可作为闪炼材料光产额测 试中的标准样品。在考虑 PMT 对 LSO:Ce 和 BGO 的不同探测效率的情况下 ${ }^{[27]}$, 通过比较 LSO:Ce 陶 瓷和 BGO 单晶各自的 ${ }^{137} \mathrm{Cs}$ 能谱图(图 10)道数可以 计算出 LSO:Ce 陶瓷的光产额为 $28600 \mathrm{ph} / \mathrm{MeV}$ 。

在 $360 \mathrm{~nm}$ 激发和 $406 \mathrm{~nm}$ 的发射监测波长下, LSO:Ce 陶瓷的发光衰减曲线如图 11 所示, 通过单 指数拟合表明该陶瓷的发光衰减时间为 $25 \mathrm{~ns}$ 。 LSO:Ce 单晶的发光衰减时间在 $30 \mathrm{~ns}$ 左右 ${ }^{[7]}$, 本实 验测得的值仍比其低 $5 \mathrm{~ns}$, 这是由于陶瓷中缺陷浓

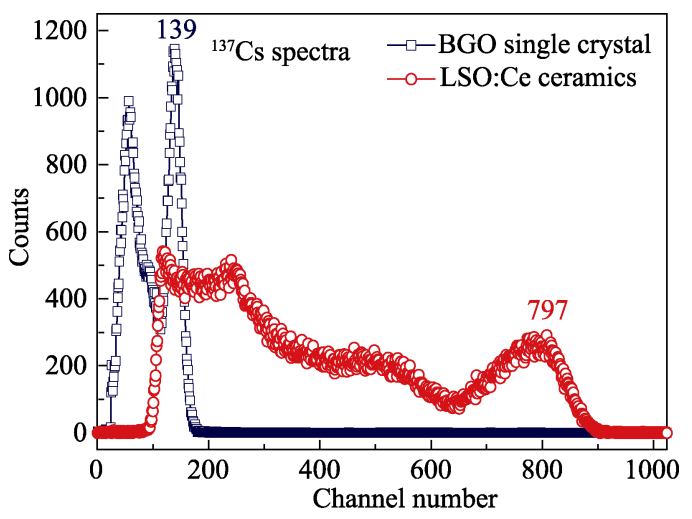

图 10 热等静压 LSO:Ce 陶瓷和 BGO 单晶的 ${ }^{137} \mathrm{Cs}$ 能谱图 Fig. $10{ }^{137} \mathrm{Cs}$ spectra of the HIPed LSO:Ce ceramics and BGO single crystal 


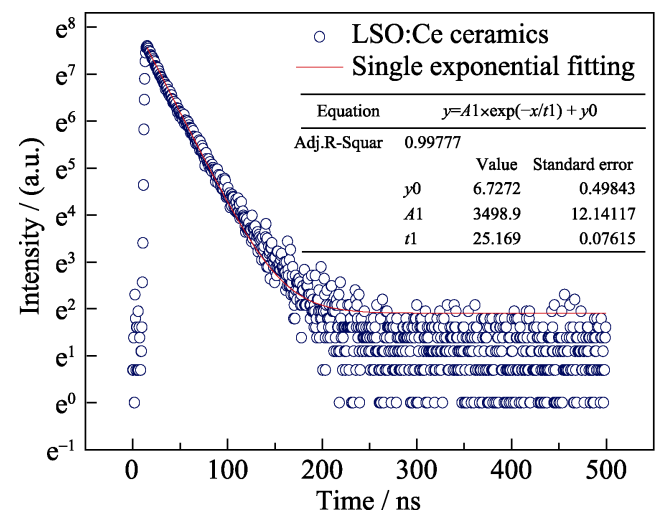

图 11 热等静压 LSO:Ce 陶瓷的发光衰减曲线 $\left(\lambda_{\mathrm{ex}}=360 \mathrm{~nm}\right.$, $\lambda_{\mathrm{em}}=406 \mathrm{~nm}$ )

Fig. 11 Luminescence decay curve of the HIPed LSO:Ce ceramics $\left(\lambda_{\mathrm{ex}}=360 \mathrm{~nm}, \lambda_{\mathrm{em}}=406 \mathrm{~nm}\right)$

度高于 LSO:Ce 单晶所致, 处于激发态的 $\mathrm{Ce}^{3+}$ 向缺 陷传递能量而发生无辐射跃迁，且无辐射跃迁过程 快于辐射跃迁过程, 缺陷浓度高会导致发光衰减时 间缩短。但是由于陶瓷中 $\mathrm{Ce}^{3+}$ 相对于 $\mathrm{Lu}^{3+}$ 的掺杂浓 度 $(0.5 \%)$ 远高于其单晶材料 (由于分凝系数限制, LSO:Ce 单晶中 $\mathrm{Ce}^{3+}$ 的掺杂浓度通常低于 $\left.0.2 \%\right)$, 在 不发生浓度淬灭的范围内, 较高的 $\mathrm{Ce}^{3+}$ 掺杂浓度会 提高 LSO:Ce 陶瓷的发光强度和光产额, 抵消或者 部分抵消由缺陷导致的发光淬灭效应。

\section{3 结论}

1) 在溶胶一凝胶法制备溶胶的基础上，采用喷 雾干燥法制备了单分散的 LSO:Ce 球形前驱粉体, 在 $1000^{\circ} \mathrm{C}$ 和 $1100^{\circ} \mathrm{C}$ 的煅烧温度下分别合成了不同结构 的 A 型 LSO 和 B 型 LSO 单相粉体。

2) 以 A 型 LSO 粉体为原料, 采用 SPS 烧结技 术在 $1200^{\circ} \mathrm{C}, 80 \mathrm{MPa}$ 的条件下保温 $5 \mathrm{~min}$ 获得相对 密度高达 99.7\%的 LSO:Ce 陶瓷, 其平均晶粒尺寸 为 $1.3 \mu \mathrm{m}$ 。

3) $\mathrm{A}$ 型球形粉体在 $1650^{\circ} \mathrm{C}$ 的空气气氛下烧结 $4 \mathrm{~h}$ 后获得了相对密度为 $98.6 \%$, 平均晶粒尺寸为 $1.6 \mu \mathrm{m}$ 的 LSO:Ce 陶瓷。该陶瓷在 $1650^{\circ} \mathrm{C} / 150 \mathrm{MPa}$ 的氩气 气氛下经热等静压(HIP)处理 $1 \mathrm{~h}$ 后, 获得了相对密 度高达 99.9\%的 LSO:Ce 半透明陶瓷，其晶粒尺寸约 为 $1.7 \mu \mathrm{m}$, 晶界干净。该陶瓷的光产额为 28600 $\mathrm{ph} / \mathrm{MeV}$, 发光衰减时间为 $25 \mathrm{~ns}$ 。

\section{参考文献:}

[1] ZHU R Y Quality of Long LSO/LYSO Crystals. Journal of Physics: Conference Series, 2012, 404(1): 012026.
[2] MELCHER C L, SCHWEITZER J S. Cerium-doped lutetium oxyorthosilicate: a fast, efficient new scintillator. IEEE Transactions on Nuclear Science, 1992, 39(4): 502-505.

[3] MELCHER C L, SCHMAND M, ERIKSSON M, et al. Scintillation properties of LSO:Ce boules. IEEE Transactions on Nuclear Science, 2000, 47(3): 965-968.

[4] BLAHUTA S, BESSI RE A, VIANA B, et al. Evidence and consequences of Ce in LYSO Ce, Ca and LYSO Ce, Mg single crystals for medical imaging applications. IEEE Transactions on Nuclear Science, 2013, 60(4): 3134-3141.

[5] MAO R, WU C, DAI L E, et al. Crystal growth and scintillation properties of LSO and LYSO crystals. Journal of Crystal Growth, 2013, 368: 97-100.

[6] YAN C, ZHAO G, HANG Y, et al. Comparison of cerium-doped $\mathrm{Lu}_{2} \mathrm{Si}_{2} \mathrm{O}_{7}$ and $\mathrm{Lu}_{2} \mathrm{SiO}_{5}$ scintillators. Journal of Crystal Growth, 2005, 281(2/3/4): 411-415.

[7] REN G, QIN L, LU S, et al. Scintillation characteristics of lutetium oxyorthosilicate $\left(\mathrm{Lu}_{2} \mathrm{SiO}_{5}: \mathrm{Ce}\right)$ crystals doped with cerium ions. Nuclear Instruments and Methods in Physics Research Section A: Accelerators, Spectrometers, Detectors and Associated Equipment. 2004, 531(3): 560-565.

[8] FARHI H, LEBBOU K, BELKAHLA S, et al. Fiber single crystal growth by LHPG technique and optical characterization of $\mathrm{Ce}^{3+}$-doped $\mathrm{Lu}_{2} \mathrm{SiO}_{5}$. Optical Materials. 2008, 30(9): 1461-1467.

[9] REN G, QIN L, LI H, et al. Investigation on defects in $\mathrm{Lu}_{2} \mathrm{SiO}_{5}$ : Ce crystals grown by Czochralski method. Crystal Research and Technology, 2006, 41(2): 163-167.

[10] QIN L, REN G, LI H, et al. Main problems in the growth of $\mathrm{Lu}_{2} \mathrm{SiO}_{5}$ :Ce scintillation crystals. Journal of The Chinese Ceramic Society, 2004, 32(11): 1361-1366.

[11] LOUTTS G B, ZAGUMENNYI A I, LAVRISHCHEV S V, et al. Czochralski growth and characterization of $\left(\mathrm{Lu}_{1-x} \mathrm{Gd}_{x}\right)_{2} \mathrm{SiO}_{5}$ single crystals for scintillators. Journal of Crystal Growth, 1997, 174: 331-336.

[12] LEMPICKI A, BRECHER C, LINGERTAT H, et al. A ceramic version of the LSO scintillator. IEEE Transactions on Nuclear Science, 2008, 55(3): 1148-1151.

[13] WANG Y, LOEF E V, RHODES W H, et al. $\mathrm{Lu}_{2} \mathrm{SiO}_{5}$ :Ce optical ceramic scintillator for PET. IEEE Transactions on Nuclear Science, 2009, 56(3): 887-891.

[14] XIE J, SHI Y, FAN L, et al. Microstructure and luminescent properties of $\mathrm{Ce}: \mathrm{Lu}_{2} \mathrm{SiO}_{5}$ ceramic scintillator by spark plasma sintering. Optical Materials, 2013, 35(4): 744-747.

[15] XIE J, LIN T, SHI Y, et al. Luminescence properties of nano-sized $\mathrm{Lu}_{2} \mathrm{SiO}_{5}$ :Ce phosphors prepared by Sol-Gel method. Journal of the Chinese Ceramic Society, 2010, 38(10): 1931- 1936.

[16] FAN L, SHI Y, XU J, et al. Consolidation of translucent $\mathrm{Ce}^{3+}$ doped $\mathrm{Lu}_{2} \mathrm{SiO}_{5}$ scintillation ceramics by pressureless sintering. Journal of Materials Research, 2014, 29(19): 2252-2259.

[17] LIN T, XU Z B, DENG L Y, et al. Spark plasma sintering of $\mathrm{Ce}^{3+}: \mathrm{Lu}_{2} \mathrm{SiO}_{5}$ scintillation ceramics and its luminescent characteristics. Journal of Inorganic Materials, 2011, 26(11): 1210-1214.

[18] LLER-BUNZ H M, SCHLEID T. On the oxide silicates $\mathrm{M}_{2} \mathrm{O}\left[\mathrm{SiO}_{4}\right]$ of the heavy lanthanides ( $\mathrm{M}=\mathrm{Dy}-\mathrm{Lu})$ with the A-type structure. 
Zeitschrift für Anorganische und Allgemeine Chemie. 1999, 625(4): 613-618.

[19] ZHU J, GU M, LIU X, et al. Phase transition and elastic and optical properties of $\mathrm{Lu}_{2} \mathrm{SiO}_{5}$. Optical Materials, 2013, 35(9): 1659-1663.

[20] FAN L, SHI Y, WU Y, et al. Crystal structure dependence of luminescence properties of Ce-doped lutetium oxyorthosilicate powder. Journal of Luminescence, 2017, 190: 504-510.

[21] GUSTAFSSON T, KLINTENBERG M, DERENZO S E, et al. $\mathrm{Lu}_{2} \mathrm{SiO}_{5}$ by single-crystal X-ray and neutron diffraction. Acta Crystallographica Section C: Crystal Structure Communications, 2001, 57(6): 668-669.

[22] WANG Y, HE Q, CHU B. Synthesis and characterization of Ce-doped $\mathrm{Lu}_{2} \mathrm{SiO}_{5}$ powders by the solid-state reaction with $\mathrm{Li}_{2} \mathrm{SO}_{4}$ flux. Journal of Alloys and Compounds, 2009, 479(1/2): 704-706.
[23] MANSUY C, MAHIOU R, NEDELEC J M. A new Sol-Gel route to $\mathrm{Lu}_{2} \mathrm{SiO}_{5}$ (LSO) scintillator: powders and thin films. Chemistry of Materials, 2003, 15(17): 3242-3244.

[24] ZYCH E, BRECHER C, WOJTOWICZ A J, et al. Luminescence properties of Ce-activated YAG optical ceramic scintillator materials. Journal of Luminescence, 1997, 75(3): 193- 203.

[25] FAN L, JIANG M, LIN D, et al. Densification of cerium-doped lutetium oxyorthosilicate scintillation ceramics by hot isostatic pressing. Journal of Alloys and Compounds, 2017, 720: 161-168.

[26] ROY S, LINGERTAT H, BRECHER C, et al. Optical properties of anisotropic polycrystalline $\mathrm{Ce}^{3+}$ activated LSO. Optical Materials, 2012, 35(5): 827-832.

[27] CHEN J, MAO R, ZHANG L, et al. Large size LSO and LYSO crystals for future high energy physics experiments. IEEE Transactions on Nuclear Science, 2007, 54(3): 718-724.

\section{我与严东生先生}

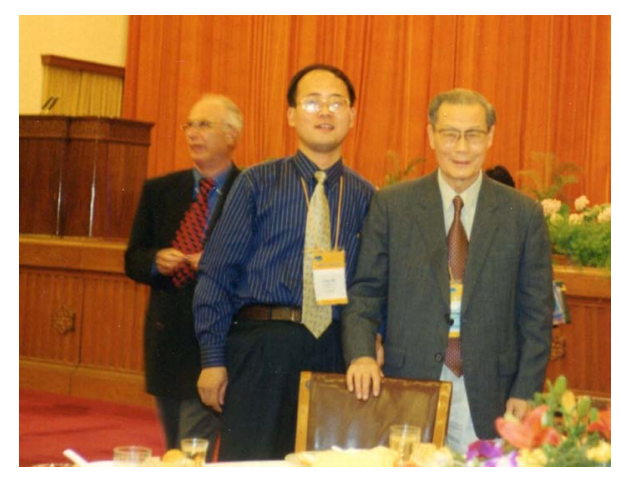

我有幸在 1992 年年底成为严先生的博士研究生。在我攻读博士 期间, 我每次呈递给严先生的工作报告和论文草稿在不长的时间内 都反馈到我手里, 让我感激不已的是, 严先生在百忙之中对我的拙 作每次都倾注心血认真修改, 大到研究方向的把握, 小到实验数据 的处理拟合, 都力求完美。对于英语文字和语法表达的修改和润色, 更是达到了其他老师难以企及的高度, 直到现在我还把严先生修改 过的两篇英文论文手稿珍藏在书柜里, 时时用来激励和鞭策自己。他 身体力行, 大力推进科研与服务于应用需求, 积极推进上硅所科研 工作服务于宝钢的产业需求, 在 1999 年北京的 IUMRS99 学术会议上他还饶有兴致地和我讨论高温钢水条 件下陶瓷部件的可靠性。

先生淡泊名利、虚怀若谷的高尚人格, 严谨求实、精益求精的工作态度, 值得我永远学习。我当永远铭 记先生 “持之以恒, 推陈出新” 的教诲, 在科研教学的道路上不断砥砺前行, 才能不辜负严先生多年的教诲 和殷切期望。 